\title{
A Review of Sensors and Their Application in Internet of Things (IOT)
}

\author{
Anukriti Sharma \\ Electronics and Communication \\ Engineering Department, \\ Maharishi Markandeshwar \\ (deemed to be University), \\ Mullana- Ambala, Haryana, India
}

\author{
Sharad Sharma \\ Electronics and Communication \\ Engineering Department, \\ Maharishi Markandeshwar \\ (deemed to be University), \\ Mullana- Ambala, Haryana, India
}

\author{
Dushyant Gupta \\ Electronics Department, Institute \\ of Honors \& Evening Studies, \\ Kurukshetra University, \\ Kurukshetra, Haryana, India
}

\begin{abstract}
Internet of Things (IOT) reflects advantages of information centric sensor network (ICSN), wireless sensor and actuator network (WSAN). The IOT becomes smarter and intelligent when combined with smart sensors. Application of sensors is one of the identified standards of technology by researchers of IOT. IOT allows communication among people and machine everywhere. The connection may employ any random path in the network and offers much application. Sensor gave new edge to IOT. A large number of sensors communicate and transfer information to serve value - added services. Sensor paves application as supply chain management, military, irrigation, aerospace, automobile, retail. Wireless sensor network (WSN) and single sensor provides smart parking, lightning, traffic management, smart water and agriculture, structural health, military, smart buildings and transport system, etc. This article presents a comprehensive review of sensors and its types and significance of sensor application in IOT through internet for the purpose of detection and retrieval of information from anywhere at any time as far as feasible.
\end{abstract}

\section{Keywords}

IOT, sensors, protocols, smart processing, data detection, data interpretation, network localization.

\section{INTRODUCTION}

Sensor, an electronic device detects the physical stimuli and converts raw data into machine or human readable form. Sensor is basically devices which can detect and respond to optical or electrical signal. It converts measured physical characteristics into electrical measurable signal. Size of sensor network depends on the sensor nodes formed. Connection amid two nodes can be done via wire or wireless. A small low power sensor node is integrated in information network. It possesses virtual personality, storing data and physical attribute using smart interface. Smart sensor nodes available at reasonable price easily provide ease to access information globally. By 2050, it is estimated that half of the world's population will reside in urban geographic location [1-3].

\subsection{Environment Monitoring}

In present scenario world is facing serious environmental issues due to brisk augment in population and pollution through industries and other sources. A catalog of few types of sensors for monitoring environment includes humidity, wind, pressure, speed, temperature, light intensity, salinity, toxic gases, and oxygen. For detecting the existing pollutants signal conditioning unit with limited potential and sensor array are employed.

\subsection{Evaluation of Environmental System}

The two prominent virtues for health care among living organism are pure water and fresh air. Pollutants are those substances when added to water alters its natural properties. Pollutants are generally categorized as pathogens which are untreated human sewage, waste from nuclear, thermal, oil or gas-based power plant, organic matter such as dead plants and animals and chemical waste from factories. Inorganic pollutants may be acidic, salt or heavy metal. Plastic food packaging consumed by living organism produces a chemical called thalate ester which has carcinogenic effect on the body. The water pollution can be categorized as non-point pollution and point pollution. The plastic food packaging when dumped in lake, sea or ocean causes a serious health hazard [4].When the water pollution occurs due to nature dumpster such as floods, pesticides sprinkled to flora termed as non- point type. Otherwise, if the pollutants added to water which may be pathogens, factory - disposal, etc. are known to cause point pollution. Addition of distinguished toxic gases such as ammonia, carbon monoxide (CO), Sulphur dioxide $\left(\mathrm{SO}_{2}\right)$, nitrogen oxide $\left(\mathrm{NO}_{2}\right)$, ozone $\left(\mathrm{O}_{3}\right)$, and particulate material (PM) to composition of air led to air pollution $[5,6]$. The expose to UV rays may cause skin cancer.

The major source of smog is waste disposal from industries and automobiles. The mixture of fog suspended in air with smoke is defined as smog. The particulate material lower than 10 micro meters in size are not visible by human naked eye when suspended in air. It causes breathing problem in humans due to blockage observed in air intake system. However, sensors need to be developed to prevent and detect particulate material, measuring air and water pollutants. Present sensor monitors presence of pollutants in the medium.

\section{SMART SENSORS}

The embedded intelligence with basic sensing mechanism is known as smart sensor [7]. Five elements for building smart sensor are sensing element, memory, interface unit, signal processor unit and software [8]. Different sensing elements detects signal for its processing, data interpretation, validity and data logging [9]. Smart sensors can accomplish access to various facilities offered by smart cities as depicted in Fig. 1. Several techniques implemented by sensors for collecting information through various sources are under continuous observation. 


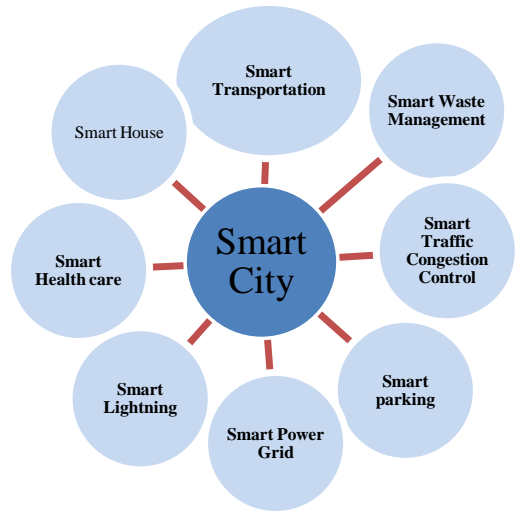

Fig1: Smart city benefits

Sensors using silicon for their development as possessed by piezo resistive, porous silicon and MEMS [6]. Sensors are fabricated via different transduction techniques as shown in Table 1 . These techniques provide an ease to fabricate, design, operate and low cost in context of sensors development. Sensors are capable in detection of electronic circuit, industries and environmental applications. The sensors are designed to provide healthy and protected life for humans, flora and fauna. Secondly, for checking the superiority of air, irrigation soil, lakes and rivers. Further, sensors reserve the natural resources for human sustainability. A systematic review is required on rainfall, volcano eruption, flush floods, soil erosion, and many more.

Table 1. Transduction techniques for sensors fabrication

\begin{tabular}{|c|c|}
\hline Sr. No. & Transduction Technique \\
\hline 1. & Micro Gravimetric \\
\hline 2. & Electro Chemical \\
\hline 3. & Electrolytic \\
\hline 4. & Optical \\
\hline 5. & Resistive \\
\hline 6. & Capacitive \\
\hline 7. & Impedance \\
\hline 8. & Piezoelectric Based Surface Acoustic Wave \\
\hline
\end{tabular}

Sensor being necessary part of IOT. The data is initially collected and then analyzed. There are some parameters which are required for various IOT applications. It includes latency, energy consumed, accuracy, etc. Some basic receiver driven approaches are discussed below for data collection framework [10].

Data driven

This approach assumes tradeoff among frequency of measured request and data accuracy. The scope for data accuracy scheme is widening when large number of sensors employed in particular geographic location.

\section{Time driven}

The elapsed time is related to the timeliness of data measurement. It may be defined as the timestamp from last measurement which is lower than maximum delay.

\section{Privacy driven}

This approach tries to maintain security issue by altering accuracy in results retrieved by individuals. The strategy involves time and data driven approaches by shortening the time duration of sensor requested for data.

Energy driven

This strategy targets to maximize gain for specific data accuracy. It is represented by utility function.

\subsection{Sensors Classification}

The end use application serves the criteria for sensors classification. Harbor Research and Postscapes investigation initially offered the initial nomenclature for sensor categorization. In this review paper sensors were arranged into different categories reported below (see Table 2.).

Table 2. Categorization of sensors

\begin{tabular}{ll}
\hline Sensor & Remark \\
\hline Smart grid & $\begin{array}{l}\text { Such sensors take care of efficient power } \\
\text { generation. }\end{array}$
\end{tabular}

Electronic This sensor gathers information from sensor equipped devices such as camera, mobile phones etc.

Chemical This sensor detects any unwanted chemical impurities in water and air.

Biosensors This class of sensors is related to animals and humans. Any sort of biological information is conveyed through these sensors.

Ambient These sensors help in examining environmental concerns.

Electric This category of sensor provides information related to electricity consumption.

Motion These sensors sensed the movement of people or things under consideration.

Position This class of sensors trace the position of any object existing globally or locally.

Identification This sensor provides a unique identity to an object for the sake of its identification.

Machine vision This sensor collected data in form of images that can be further processed by using computers.

Load/Force The load/force sensor recorded the deformation observed by any system due to application of load on it.

Hydraulic These sensors are used for determining and controlling the flow rate of liquid generally water.

Presence These sensors are used in security system for the purpose of identifying the presence of any unwanted thing in an unauthorized area.

Acoustic Such sensors monitoring the change in 
sound level observed in surroundings.

Interaction

Such sensors examine the human behavior from outside and act accordingly. Sliders and buttons fall under this category of sensor.

\subsubsection{Smart Grid Sensors}

These sensors guarantee power generation, its transmission and distribution efficiently from source to end users. A smart grid technique is categorized into five segments depending upon their role, sensors, component advancement, communication and supporting decision system [4].

Electronics sensor. Such sensors sense the stored data from variety of sensor including mobile phone, security street camera, etc. The analyzed data serve a welfare state of living to citizen in terms of security, level of accuracy in generating data and transmitting data [11]. Detection of various forms of energy via electroscope, voltage detection, magnetic anomalies, etc. is the major functionality performed by these sensors. In smart urban, sensors when combined with neural network develop and undergo analyzing data from image, speech recognition, natural language processing and video [12].

\subsubsection{Chemical sensor}

These sensors identify the presence of chemical composition in the air and problem linked to it. Such class of sensor includes oxygen sensors, $\mathrm{pH}$ sensor, gas sensor, smoke detector sensor, carbon dioxide sensors, catalytic bead sensors, electronic nose, etc. Chemical sensor can sense the physical and chemical properties of a system. It is implacable in medical field to diagnose allergy in living body [13].

\subsubsection{Biosensor}

These sensors work in the field of bio medicine. Neutron and MEMS sensors are utilized for ionizing and subatomic sensors. It works on the principle of transduction and adopt optical techniques for attaining accuracy at a fast pace. Measurement of non- polar molecules, anomalies could be detected via electro chemical sensors $[14,15]$. Concentrations of acolyte are shown by electrical properties and chemical property of ions. These are either wearable or could be implanted on /in living body so as to collect data of particular subject representing biological information. Biosensors include heartbeat and breathe sensor, body posture sensor, sensor for elderly and kids, etc. Biosensors are of four types as illustrated in Fig. 2.

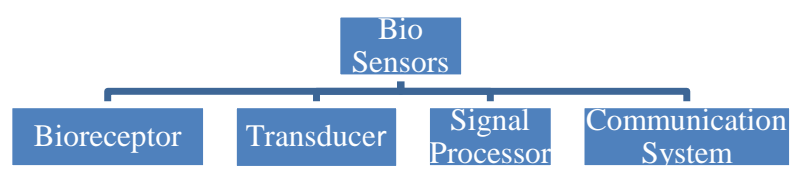

Fig 2: Biosensors Classification

Bioreceptor also called as recognition element. It senses biological characteristics in living body. Different types of bio receptor include anti body, enzyme, antigen, protein, etc.
Transducer converts particular characteristics into equivalent electrical signal. Different types of transducer include optical, calorimeter, electromechanical, mass change, etc. Signal processor blocks filters noise using finite impulse response filter (FIR). Amplification is performed to enhance the strength of signal so as to display or transmit for storing and analyzing.

\subsubsection{Ambient sensor}

This sensor is combination of different sensors likewise temperature, atmospheric pressure, light, humidity sensor. The collected data from sensor helps in monitoring environmental issues.

\subsubsection{Motion sensor}

Motion sensor detects the movement of living being or objects. Two vital components included are gyroscope and accelerometers. The range of variation of axis from 3 up to 9 axes.

\subsubsection{Electric sensor}

These are multi variant data provider where parameters such as tension, current and electricity related data is sensed. Electric sensor could monitor how much energy is consumed at a site.

\subsubsection{Identification sensor}

These sensors are capable to identify any object to the system. The cards or tags have utility for string semantic purpose. Radio frequency identification (RFID) and near field communication (NFC) are the elements of IOT and modern identifying techniques.

\subsubsection{Position sensor}

These sensors locate the position of object which may globally or referred locally. Global positioning space (GPS) sensor provides data spatial information covering $2 \mathrm{G}$ coordinates to complex collection of data. Examples of position sensors are magnetometers, fixed wireless network locationization service, GPS via received signal strength (RSS) processing information.

\subsubsection{Presence sensor}

These are passive infrared sensor (PIR). They can detect presence of living organism in particular authorized area. It is implemented in security systems and many more.

\subsubsection{Machine vision sensor}

The vision based sensor collect data and forward for utilization of computer aided/ assisted vision to serve IOT platform. They have applicability in context of both conventional and infrared security cameras. This kind of sensor employs the image processing techniques for sensing the detection of movement of human or entities.

\subsubsection{Interaction sensor}


These sensors classified under interaction sensor have pipelining for cognizant living organism interaction. They observe human behavior from surroundings and respond accordingly. Sliders and buttons are illustrations under classification of such sensors.

\subsubsection{Acoustic sensor}

These sounds activated gadgets collect the data of sound wave to forward same to respective application. The examples of devices which implement sound wave are piezoelectric sensor and microphones.

\subsubsection{Force sensor}

Speedometer sensor and load sensor are gathered into category of force sensors. It is the measure of force applied to them externally.

\subsubsection{Hydraulic sensor}

Water quality which monitors and measure the liquid level, its flow intensity and properties are termed as hydraulic sensors. It includes water and other liquids.

\subsubsection{Object information sensors}

This sensor falls into category of context application sensor. It provides data about any entity.

\section{PRESENT STATUS}

Morais et al. [16] contributed in building up understanding of dynamic IOT to data researchers by plotting the IOT featuring seven prominent scenario's with particular description of its variables, quantifier and sensors organization. Secondly, recognition of nineteen data types has been accomplished. Amir Badshah et al. [17] explored the procedure to navigate transport unit in smart cities using implementation of vision based sensor and advanced normalized phase correlation. Thus, avoids deploying (GPS) Global Positioning System as well as calibrated sensors. Such mapping of vehicles requires image registration. The investigator successfully got accurate result in comparison to calculated data from GPS corresponding to estimated position accuracy. Gao chong et al. [18] examined the athlete and comeback of smart home system based on browse/ server (B/S) module. The architecture of purposed smart home system along with its hardware design and its implementation criteria has been explained. The user can remotely control the house hold objects. The outline performance of the above-mentioned system is flexible and beneficial. Soumya Basak et al. [19] introduced (RMS) Remote Monitoring Station. It is achieved when interconnection of internet with wireless network, (MQTT) Message Queuing Telemetry Tracking is established, the sensors and CC 3200 launch pad by Texas Instrument. The application lies in providing climatic alerts to farmer helping them in growth and maintenance of crop production. A.R. Ali et al. [20] discussed the architecture of wireless smart sensors, its associated standards, protocols, network topologies and information regarding its implementation. The mobility setup in network of wireless smart sensors via variety of protocols likewise IEEE 1451. B. Soh et al. [12] explored the element responsible for setting up smart city that is 'network sensors'. The authors proposed variety of solutions and ethical implications to address challenges faced during evolution of new techniques. It includes delivery of service \& optimization, security \& safety management, traffic control \& parking, smart building, public transport and many more. Yaw-Wen kuo et al. [21] focused on the application of IOT offered to daily routine of users applied in the automation system through environment sensor. Due to lack of availability in transmission of electricity, low transmission and long range of power is required so that sensor node draw less amount of current during transmission. The role of (MAC) media access control and RF radio technologies has been discussed. The author designed commercial module based sensor node which implemented IEEE 802.15.4e $(\mathrm{TaSCH})$ time slotted channel hopping. Alcaya et al. [22] explored the detailed architecture for angle of attack sensors and mentioned brief description of its drawing. A water management system for angle of attack sensor and issues related with sensor has been focused. Himadri Nath Saha et al. [23] surveyed literature on disaster management on risk identified during disaster and its preparedness. The phenomena of disaster management includes emergency response, allocation of resources, reaction planning and ends up at recovery of disaster using early warning, sensors and IOT standard. KamanashisBis et al. [24]studied three chief factor of sensor nodes as energy with associated position and supremacy of link when kept inside or outside the network by deploying numerous base station performing simulations in mat lab and calculated life span of node by applying one of the routing practices. Xuxun Liu et al. [25] implemented (ACO) ant colony optimization. Researcher developed scheme of global optimal distance acquirement by adopting the process of network lifetime estimation and accomplished with the objective of high energy consumed throughput network and low energy dissipation from sensor nodes. Wenzhengxu et al. [26] reconnoitered when nodes are partially charged through convey of energy wirelessly by implementing magnetic resonant coupling. Canvasser compared state of art benchmark with the outcome of entire space travelled by mobile charger 1 to $15 \%$ elongated and maximum total sensors lifetime is $9 \%$ with reverence to average energy expiration duration per sensor by means of two types of algorithms. Loizos Kanaris et al. [27] verified and compared the remarks of simulation performed during designing of IOT networks. In this experiment, connection of two simulators as done with the network layer and 3D polymetric radio propagation. The author proposed methodology in flowchart form in case of TruNET- Cooja interconnection. Tao Liu et al. [28] explored the structure of IOT technologies and implementation of its functionalities. The application of IOT serving in clinical care and real time ECG monitoring using telemedicine technology has been discussed. Henrich C. Pohls et al. [29] implementation of software based ECC on constrained devices for secure digital signature. ECDSA p 160 NIST is the software prototype. The aim is to reconstruct the previous signed bit representation. Soumya Kanti Datta et al. [30] concluded the feasibility of integrated semantics computing on android powered mobile devices. Discussion for embedding M3 framework with its light weight version in mobile application has been done. Thus, architecture of machine to machine and its associated prototype has been implemented. Prahlada Rao B. B et al. [31] explored the project on working of cloud computing and IOT for addressing big data issues. The various applications such as irrigation, environmental monitoring and augmented reality are realized on cloud using sensor as service. Qian Zhu et al. [32] implemented IOT gateway working as bridge between network of network and wireless sensor network. 
The gateway based on Zig bee- GPRS protocols. The author finds application in smart city, industrial and environmental monitoring. Uday Shankar Shanthamallu et al. [33] presented brief review on conceptual machine learning and applicability of its algorithms. A variety of modals including supervised, unsupervised methods and deep learning processes has been focused upon. In the paper, it was mentioned that Galaxy S5 has inbuilt 26 number of different sensors, namely, proximity, camera, pressure, humidity, gyro, microphone, magnetometer, accelerometer, infra-red, etc. The applications of algorithm are utilized for anomaly detection, sensor networking, and pattern recognition and at different layers of IOT architecture. S.S.Navghane et al. [34] designed sketch for implementing IOT based smart dustbins together with Wi-Fi module, weight and IR sensors for contributing hygiene in the society. The current status of garbage could be retrieved on mobile web browser via Wi-Fi with html page. Interfacing of microcontroller possessing IR sensor with central system is done using Wi-Fi module. Khirod Chandra Sahoo et al. [35] analyzed intruder detection in order to maintain security. The author discussed the application and characteristics of (PIR) passive infrared sensor capable of detecting the anomalous motion even in dark surroundings. Zig bee is used for creating wireless sensor network and ESP 8266 module to transmit data to a remote server. The global system for mobile communication (GSM) sends text alerts to respective authorities regarding detection of intruder. All sensor nodes connected to center node utilize Zig bee for transmitting and receiving data wirelessly.

\section{SENSOR BASED IOT APPLICATIONS \\ 4.1 Clinical Care}

In case of emergency to patient, certain parameters such as blood pressure, glucose level, heart condition are necessary to monitor prior to prescribing medicine. Only sensor based IOT for healthcare could provide fast access to these test. It provides remote monitoring of patients. An electronic sensor when connected to patient could retrieve information of chemical imbalance in physical, psychological and behavior. The information regarding patient from any location is made available to medical practitioner around the clock via IOT devices wirelessly. It enables doctors for recommendations distantly.

\subsection{Smart City}

The rising population curve is the critical reason for the requirement of smart cities. Urban dynamics like water, land, health, etc could be improved sustainably and efficiently [36]. Thermal sensors track transmission and distribution path of energy with regard to changing weather conditions [37]. The quality of service (QoS) provided in cities turning them smarter is only possible with advancement in cloud computing and fusion of smart sensors in IOT devices [15]. A blend of advanced sensors for storing information and analyzing it for communication so as to manage cities assets brought up concept of smart city [38]. A smart grid technique is categorized into five segments depending upon their role, sensors, component advancement, communication, supporting decision system, etc. [39]. The application of IOT plays vital role for implementing and conceptualizing smart city where sensor nodes are deployed for connectivity [12]. The public service has been revolutionary served with the advancement of smart sensors with IOT devices in cities [40]. Applications of smart city are presented with adoption of digitization program by government around the globe. It is responsible for policy and infrastructure layout of smart city [41].

\subsection{IOT and Medical Robotics}

Robots are programmed machines so as to accomplish respective task in distinguish fields. Such as healthcare, industrial, etc. Smart sensor combined to IOT devices provides input to the robots. The obtained characteristics are sent to server of healthcare centre using protocols like Bluetooth, zig bee, wireless fidelity (Wi-Fi), etc.

\subsection{Smart Home}

Smart home include control by any of the communication equipment and display via all kinds of interfaces[42].Home gateway fixes different interfaces and covers variety of communication techniques. Features of smart home are as follows: firstly, compatible to different communication technologies. Secondly, fetching information about smart home becomes convenient as it provides ubiquitous service. Lastly, comprehensive perception serves many logical and physical sensors monitors real time smart home. The architecture model relies on IOT for a smart home is depicted in Fig. 3.
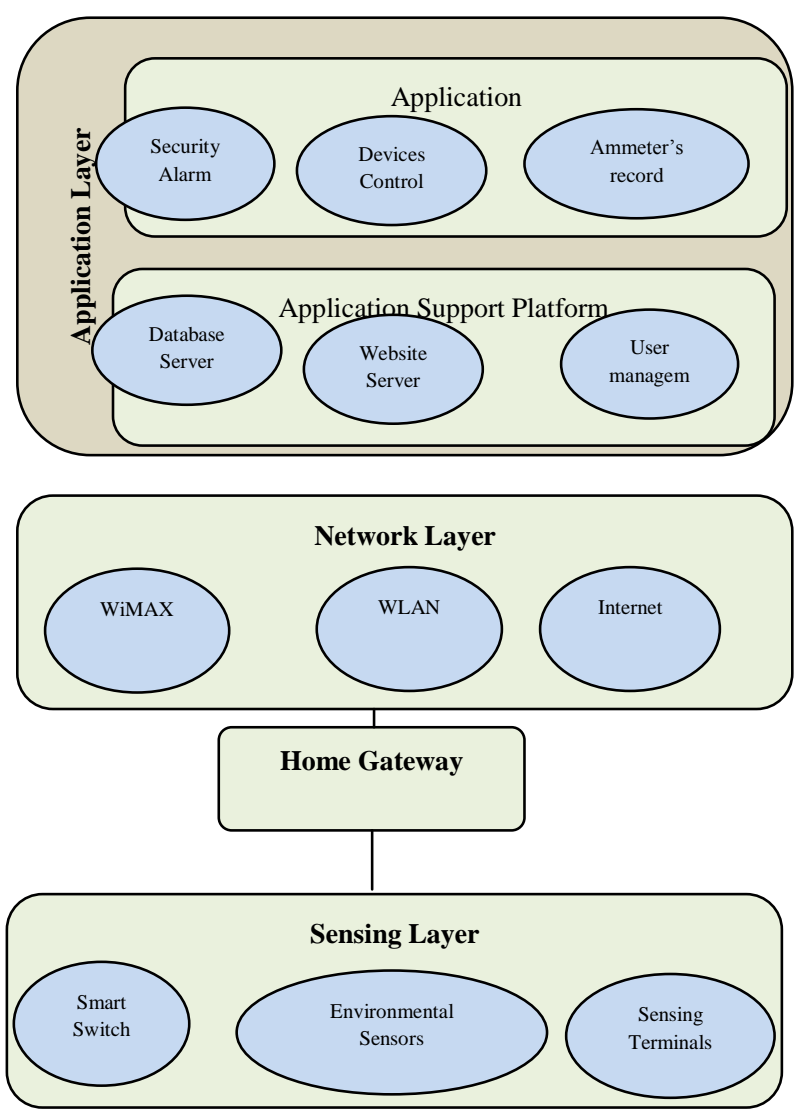

Fig.3: Smart home architecture model

\subsubsection{Sensing layer}

Large number of physical and logical sensors monitors household objects. Some of the sensing terminal includes environment monitor sensor, camera, GPS, household object controlling sensor, etc. Short range wireless, Zig bee, sensing technology finds broader application in smart home due to its 
reflecting features of low complexity, cost and power [43]. The meshing architecture is used for composing wireless sensor network (WSN).

\subsubsection{Home gateway}

This is connector among perception and network layer. It allows transmission of information due to its compatibility with various communication interfaces. It is also called 'Protocol conversion unit'.

\subsubsection{Network layer}

Perception layer transmits information for processing network layer via home gateway. Hybrid network integration technique provides information access to each network. Examples of wireless and wired communication are mobile cellular network, internet, wireless local area network (WLAN).

\subsubsection{Application layer}

This layer contains concrete application support platform. It acts as interface among users and smart home system.

\subsection{Smart Parking}

Due to increase in number of vehicles, parking has emerged as crucial component in smart transport system. Improper parking results in traffic congestion. Parking done in unauthorized area led to mismanagement in transport system. Sensors could be deployed in parking location. Information regarding availability of parking slot could be fetched by users through application in android smart phone.

Earlier investigations on vehicular traffic unfolds that about $30 \%$ of traffic congestion occurs due to parking of vehicles done according to connivance of driver [44]. The advent of smart parking provides optimization in terms of fuel consumption, manual efforts and time savings.

The investigators have implemented ultrasonic and infrared sensors, Raspberry Pi3 board for smart parking using IOT. The information detected by sensors is stored in cloud using a processing module. The status of vacant parking space could be retrieved by user through android smart phone application. The block diagram of WSN based smart parking application using IOT is represented in Fig. 4.

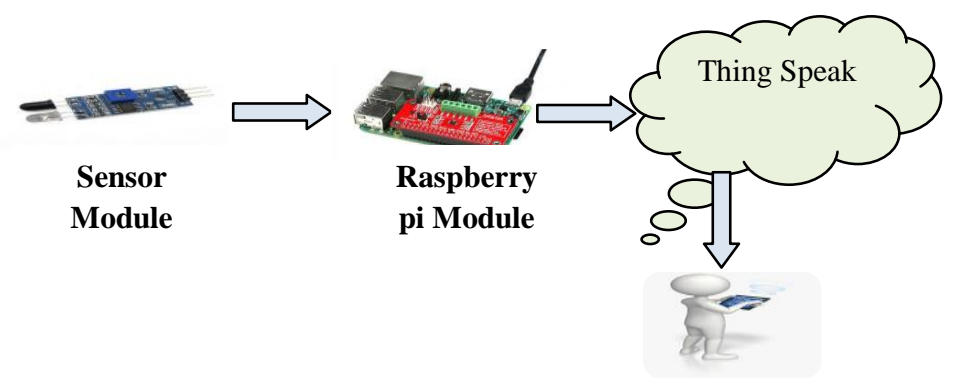

Mobile User

Fig.4: Block diagram
A sensor node is deployed at every parking slot. Ultrasonic and infrared sensors are responsible for updating information regarding vacant status for parking.

\subsubsection{Raspberry Pi module}

It is used as System on Chip (SOC). It is lower cost computer with smaller size as that of credit card.

\subsubsection{Thing Speak}

Thing Speak is a web service related to IOT, developed for the purpose of saving sensed data of various IOT based applications. Further, it assists in plotting the output data in graphical form [45].

\subsubsection{Mobile user}

All tablet or smart phone users are mobile users whether they use them while traveling or put these gadgets statically.

\subsection{Smart irrigation [19]}

In order to achieve the target of ideal yield from the fields, smart agriculture has been adopted. The message queuing telemetry tracking (MQTT) protocol, wireless networking is combined with internet of things (IOT) so as to monitor the real time field environment. Data from field at any instant of time could be collected by Sensors, MQTT and CC3200 launch pad by Texas Instrument. After that, it is sent to remote monitoring station (RMS).

Factors influencing growth of crop can be monitored likewise soil fertility level, water management, sunlight intensity, dosage of fertilizers, altering climatic conditions- humidity and temperature. Components deployed for the test setup are discussed below.

\subsubsection{Light Detection Resistor (LDR)}

It is photosensitive device to detect sunlight intensity. The resistance is very low in case of exposure to light and vice versa.

\subsubsection{Soil Moisture Sensor}

Soil sensor is useful to detect the content of moisture in soil. The module output is at lower level at times when excess water present in soil. Sensor operates on $20 \mathrm{~mA}, 5 \mathrm{~V}$.

\subsubsection{Water Pump}

The operating range of water pump is 6 to $9 \mathrm{~V}$ DC. Its functionality is to suck water via a nozzle and pump it out with larger force, through another nozzle.

\subsubsection{Four channel relay module}

It works at range of $5 \mathrm{~V}, 10 \mathrm{~A}$. Relay may act as amplifier or switch. It amplifies smaller value of current generated by sensor. 
Texas Instrument developed first CC3200 Launch pad on chip internet. The Wi-Fi interfacing connection $802.11 \mathrm{~b} / \mathrm{g} / \mathrm{n}$ at low power consumption. Wi-Fi unit board is compatible with MQTT \& HTTP protocols. At rate of $80 \mathrm{MHz}$, micro controller unit also possess 32 bit advanced RISC machinecortex M4.

\subsubsection{Motor}

It converts electrical energy into mechanical energy. In comparison to normal dc motor, greater torque and a greater number of rotations per minute (RPM) is provided by $\mathrm{L}$ shaped motor. It has 300 RPM.

\section{CONCLUSION AND FUTURE WORK}

The innovated devices possess the capabilities to actuate and communicate with other devices which brought adaptability to the concept of Internet of Things, here the role of actuator and sensor play incalculably in the environment and new-fangled capabilities are accessible through vast information resources. The IoT is an upcoming technology for creating revolutionary application domain of Plug n Play smart devices. The future research work relies on utility application by 2020 and transport field by 2025 and thereafter.

The former includes energy production and its recycling, large scale WSN, high protection, deploying and forgetting networks, self-adaption system of system, cloud computing and online analysis with data storage, renewable material and nano power units, monitoring of critical infrastructure, smart grid and housing metering. Furthermore, a lot of research work is required for transportation under application domain. These are enlisted as smart tags for automobile management and logistics, motor vehicle to infrastructure, system level analytics, and autonomous means of transportation using IOT services, heterogeneous systems with interaction between other sub network, smart traffic, automatically driven vehicles, intelligent transport and logistics.

\section{REFERENCES}

[1] Babar, M.A., L. Zhu, and R. Jeffery. A framework for classifying and comparing software architecture evaluation methods. in 2004 Australian Software Engineering Conference. Proceedings. 2004. IEEE.

[2] Karakostas, B., A DNS architecture for the internet of things: A case study in transport logistics. Procedia Computer Science, 2013. 19: p. 594-601.

[3] Thoma, M., et al. On iot-services: Survey, classification and enterprise integration. in 2012 IEEE International Conference on Green Computing and Communications. 2012. IEEE.

[4] Easterbrook, S., et al., Selecting empirical methods for software engineering research, in Guide to advanced empirical software engineering. 2008, Springer. p. 285311.

[5] Bergerman, M., C. Lee, and Y. Xu. Dynamic coupling of underactuated manipulators. in Proceedings of International Conference on Control Applications. 1995. IEEE.

[6] Tory, M. and T. Moller. Rethinking visualization: A high-level taxonomy. in IEEE Symposium on Information Visualization. 2004. IEEE.
[7] Arriaga, R.I., et al., Ubiquitous wearable electrochemical sensors. The Electrochemical Society Interface, 2016. 25(4): p. 69-72.

[8] Mondal, S.P., et al., Development of high sensitivity potentiometric NOx sensor and its application to breath analysis. Sensors and Actuators B: Chemical, 2011. 158(1): p. 292-298.

[9] Pramanik, P.K.D., et al., Internet of things, smart sensors, and pervasive systems: Enabling connected and pervasive healthcare, in Healthcare Data Analytics and Management. 2019, Elsevier. p. 1-58.

[10] Siris, V.A., et al., Smart application-aware IoT data collection. Journal of Reliable Intelligent Environments, 2019. 5(1): p. 17-28.

[11] Gu, C. and J. Lien, A two-tone radar sensor for concurrent detection of absolute distance and relative movement for gesture sensing. IEEE sensors letters, 2017. 1(3): p. 1-4.

[12] Alharbi, N. and B. Soh. Roles and Challenges of Network Sensors in Smart Cities. in IOP Conference Series: Earth and Environmental Science. 2019. IOP Publishing.

[13] Saidi, T., et al. Detection of seasonal allergic rhinitis from exhaled breath VOCs using an electronic nose based on an array of chemical sensors. in 2015 IEEE SENSORS. 2015. IEEE

[14] Pospíšilová, M., G. Kuncová, and J. Trögl, Fiber-optic chemical sensors and fiber-optic bio-sensors. Sensors, 2015. 15(10): p. 25208-25259.

[15] Wang, J., et al., Vehicular sensing networks in a smart city: Principles, technologies and applications. IEEE Wireless Communications, 2017. 25(1): p. 122-132.

[16] de Morais, C.M., D. Sadok, and J. Kelner, An IoT sensor and scenario survey for data researchers. Journal of the Brazilian Computer Society, 2019. 25(1): p. 4.

[17] Badshah, A., et al., Vehicle navigation in GPS denied environment for smart cities using vision sensors. Computers, Environment and Urban Systems, 2019. 77: p. 101281.

[18] Chong, G., L. Zhihao, and Y. Yifeng. The research and implement of smart home system based on internet of things. in 2011 International Conference on Electronics, Communications and Control (ICECC). 2011. IEEE.

[19] Mukherji, S.V., et al. Smart Agriculture using Internet of Things and MQTT Protocol. in 2019 International Conference on Machine Learning, Big Data, Cloud and Parallel Computing (COMITCon). 2019. IEEE.

[20] Al-Ali, A., et al. Wireless smart sensors networks overview. in Second IFIP International Conference on Wireless and Optical Communications Networks, 2005. WOCN 2005. 2005. IEEE.

[21] Kuo, Y.-W. and C.-L. Li. Design of long range low power sensor node for the last mile of IoT. in 2016 IEEE International Conference on Consumer ElectronicsTaiwan (ICCE-TW). 2016. IEEE.

[22] Formhals, A., United States: Patent Application Publication. US patent, 1934. 1(975): p. 504 
[23] Saha, H.N., et al. Disaster management using Internet of Things. in 2017 8th Annual Industrial Automation and Electromechanical Engineering Conference (IEMECON). 2017. IEEE.

[24] Biswas, K., et al., An analytical model for lifetime estimation of wireless sensor networks. IEEE Communications Letters, 2015. 19(9): p. 1584-1587.

[25] Liu, X., An optimal-distance-based transmission strategy for lifetime maximization of wireless sensor networks. IEEE Sensors Journal, 2014. 15(6): p. 3484-3491.

[26] $\mathrm{Xu}, \mathrm{W}$., et al., Maximizing sensor lifetime with the minimal service cost of a mobile charger in wireless sensor networks. IEEE Transactions on Mobile Computing, 2018. 17(11): p. 2564-2577.

[27] Kanaris, L., et al., On the realistic radio and network planning of iot sensor networks. Sensors, 2019. 19(15): p. 3264.

[28] Lu, D. and T. Liu. The application of IOT in medical system. in 2011 IEEE International Symposium on IT in Medicine and Education. 2011. IEEE.

[29] Pöhls, H.C. JSON Sensor Signatures (JSS): end-to-end integrity protection from constrained device to IoT application. in 2015 9th International Conference on Innovative Mobile and Internet Services in Ubiquitous Computing. 2015. IEEE.

[30] Datta, S.K., et al. oneM2M architecture based user centric IoT application development. in 2015 3rd International Conference on Future Internet of Things and Cloud. 2015. IEEE.

[31] Rao, B.P., et al. Cloud computing for Internet of Things \& sensing based applications. in 2012 Sixth International Conference on Sensing Technology (ICST). 2012. IEEE.

[32] Zhu, Q., et al. Iot gateway: Bridgingwireless sensor networks into internet of things. in 2010 IEEE/IFIP International Conference on Embedded and Ubiquitous Computing. 2010. Ieee.

[33] Shanthamallu, U.S., et al. A brief survey of machine learning methods and their sensor and IoT applications. in 2017 8th International Conference on Information, Intelligence, Systems \& Applications (IISA). 2017. IEEE.

[34] Navghane, S., M. Killedar, and V. Rohokale, IoT based smart garbage and waste collection bin. International Journal of Advanced Research in Electronics and
Communication Engineering (IJARECE), 2016. 5(5): p. 1576-1578.

[35] Sahoo, K.C. and U.C. Pati. IoT based intrusion detection system using PIR sensor. in 2017 2nd IEEE International Conference on Recent Trends in Electronics, Information \& Communication Technology (RTEICT). 2017. IEEE.

[36] Riva Sanseverino, E., R. Riva Sanseverino, and E. Anello, A cross-reading approach to smart city: A european perspective of chinese smart cities. Smart Cities, 2018. 1(1): p. 26-52.

[37] Jiang, J.-A., et al., A novel weather information-based optimization algorithm for thermal sensor placement in smart grid. IEEE Transactions on Smart Grid, 2016. 9(2): p. $911-922$.

[38] Csáji, B.C., et al., Wireless multi-sensor networks for smart cities: A prototype system with statistical data analysis. IEEE Sensors Journal, 2017. 17(23): p. 76677676.

[39] Larik, R.M., M.W. Mustafa, and S.H. Qazi, Research Article Smart Grid Technologies in Power Systems: An Overview. Research Journal of Applied Sciences, Engineering and Technology, 2015. 11(6): p. 633-638.

[40] Morello, R., et al., Advances on sensing technologies for smart cities and power grids: A review. IEEE Sensors Journal, 2017. 17(23): p. 7596-7610.

[41] Li, W., H. Song, and F. Zeng, Policy-based secure and trustworthy sensing for internet of things in smart cities. IEEE Internet of Things Journal, 2017. 5(2): p. 716-723.

[42] Liu, Y., Study on smart home system based on internet of things technology, in Informatics and Management Science IV. 2013, Springer. p. 73-81.

[43] Park, H., J. Burke, and M.B. Srivastava, Intelligent Lighting Control using Wireless Sensor Networks for Media Production. KSII Transactions on Internet \& Information Systems, 2009. 3(5).

[44] Balhwan, S., D. Gupta, and S. Reddy. Smart Parking-A Wireless Sensor Networks Application Using IoT. in Proceedings of 2nd International Conference on Communication, Computing and Networking. 2019. Springer.

[45] Pasha, S., ThingSpeak based sensing and monitoring system for IoT with Matlab Analysis. International Journal of New Technology and Research, 2016. 2(6). 